УДК 532.536

\title{
НЕУСТОЙЧИВОСТЬ ПОТОКА В ПОРИСТОМ МИКРОКАНАЛЕ
}

\author{
Авраменко А.А., член-корреспондент НАН Украины, Дмитренко Н.П., канд. техн. наук, \\ Ковецкая Ю.Ю.
}

Институт технической теплофизики НАН Украины, ул. Желябова, 2а, Киев, 03680, Украина

Досліджена нестійкість течії в плоскому пористому мікроканалі. Використано метод колокації для чисельного знаходження критичних чисел Рейнольдса, які визначають область гідродинамічної нестійкості потоку. Також виявлено вплив пористості та проковзування на критичні хвильові числа.
Исследована неустойчивость течения в плоском пористом микроканале. Использован метод колокации для численного определения критических чисел Рейнольдса, которые определяют область гидродинамической неустойчивости потока. Также определено влияние пористости и проскальзывания на критические волновые числа.
The flow instability in a flat porous microchannel is investigated. The collocation method for the numerical determination of the critical Reynolds number, which determines the hydrodynamic flow instability, is used. Also the effect of porosity and slipping on the critical wave numbers is determined.

Библ. 10, рис. 3.

Ключевые слова: неустойчивость, пористость, проницаемость, поток с проскальзыванием, волновое число.

$h$ - ширина канала;

$L$ - длина свободного пробега;

$\widetilde{p}$ - давление;

$K$ - проницаемость;

$\widetilde{P}$ - давление основного потока;

$U$ - безразмерная скорость основного потока;

$\widetilde{U}-$ скорость основного потока;

$\tilde{u}$ - компонента скорости для $\tilde{x}$;

$\tilde{v}$ - компонента скорости для $\tilde{y}$;

$\tilde{x}, \tilde{y}$ - декартовые координаты;

\section{Введение}

Исследования физики процессов, протекающих на уровне микромасштабов в пористых средах с разреженными потоками, в последнее время привлекает большой интерес. Это связано со стремлением к миниатюризации бытовых и промышленных устройств, а также с уникальными характеристиками, присущими физическим явлениям на микромасштабах.

Исследования динамики потока с пористой средой на микромасштабах проводятся по следующим направлениям [1-3]:

- охлаждение электроники с использованием микроустройств (микроканалы, микродатчики, химические микрореакторы);

- биомедицинские исследования;

- микро-электромеханические системы;

- выпаривание различных веществ;

- интенсификация теплопереноса и многое другое.

Существует много работ по гидродинамической неустойчивости в микроканалах различной геометрии при течении чистой среды $[4,5]$. Процессы неустойчивости ламинарного течения в пористой среде, заполненной разреженным газом, изучены недостаточно хорошо. Впервые неустойчивость ламинарного течения в канале, заполненном пористой средой, была исследована $\tilde{\alpha}$ - волновое число возмущений вдоль потока;

$\widetilde{\beta}$ - волновое число для поперечной координаты;

$\rho-$ плотность;

$v$ - кинематическая вязкость.

Безразмерные комплексы:

$$
\begin{aligned}
& M=(\mathrm{Da})^{-1 / 2} ; \mathrm{Da}=\frac{K}{\widetilde{h}^{2}}, \operatorname{Re}=\frac{\widetilde{u}_{\infty} \widetilde{h}}{v} ; \\
& \alpha=\widetilde{\alpha} \widetilde{h} ; U=\frac{\widetilde{u}}{u_{\infty}} ; c=\frac{\widetilde{\beta}}{\widetilde{\alpha} \widetilde{u}_{\infty}} ; \varphi=\frac{\widetilde{\varphi}}{u_{\infty} h} .
\end{aligned}
$$

Нилдом в [6]. В указанной работе была использована аналогия между потоком в пористой среде и магнитным гидродинамическим течением. Авторы [7] продолжили работу [6] и получили модифицированное уравнение Орра-Зомерфельда, которое учитывает линейную составляющую гидродинамического сопротивления (Дарси) с проскальзыванием.

В работе [8] авторы исследовали влияние проскальзывания на устойчивость ламинарного потока в плоском канале. Была получена зависимость критического числа Рейнольдса от числа Кнудсена.

Как известно, эффекты разрежения в потоках характеризуются числом Кнудсена (Kn) (Knudsen), которое является безразмерным параметром, пропорциональным отношению между средней длиной свободного пробега молекул газа $(L)$ и расстоянием между стенками канала.

В настоящей статье исследуется устойчивость ламинарного течения в плоском микроканале, ширина которого $2 h$. Канал заполнен пористой средой. Движение потока обеспечивается продольным градиентом давления. Рабочим телом является разреженный газ. Согласно [9] при $10^{-3} \leq \mathrm{Kn} \leq 10^{-1}$ возникает так называемый режим с проскальзыванием, где действует допущение континуума. 
В пористых средах поток может носить как ламинарный, так и турбулентный характер. И важно знать условия и параметры, при которых один режим течения переходит в другой. С целью определения критерия неустойчивости рассмотрим особенности динамики потока с проскальзыванием в пористой среде, используя двумерную постановку задачи.

\section{Основные уравнения}

Динамика потока в пористой среде описывается модифицированным уравнением Навье-Стокса и уравнением неразрывности. Уравнение движения отличается от классического [10] тем, что оно содержит член, учитывающий линейное сопротивление Дарси.

$$
\begin{aligned}
& \frac{\partial \widetilde{u}}{\partial \widetilde{t}}+\widetilde{u} \frac{\partial \widetilde{u}}{\partial \widetilde{x}}+\widetilde{v} \frac{\partial \widetilde{u}}{\partial \widetilde{y}}=-\frac{1}{\rho} \frac{\partial \widetilde{p}}{\partial \widetilde{x}}+v \nabla^{2} \widetilde{u}-\frac{v}{K} \widetilde{u}, \\
& \frac{\partial \widetilde{v}}{\partial \widetilde{t}}+\widetilde{u} \frac{\partial \widetilde{v}}{\partial \widetilde{x}}+\widetilde{v} \frac{\partial \widetilde{v}}{\partial \widetilde{y}}=-\frac{1}{\rho} \frac{\partial \widetilde{p}}{\partial \widetilde{y}}+v \nabla^{2} \widetilde{v}-\frac{v}{K} \widetilde{v}, \\
& \frac{\partial \widetilde{u}}{\partial \widetilde{x}}+\frac{\partial \widetilde{v}}{\partial \widetilde{y}}=0,
\end{aligned}
$$

где волна указывает на то, что параметр размерный.

Используем метод малых линейных возмущений для определения критериев гидродинамической неустойчивости разреженной среды. Соответственно этому методу основные параметры течения представим в виде суммы основных (невозмущенных) и малых (возмущающих) параметров.

$\widetilde{u}=\widetilde{U}(\widetilde{y})+u^{\prime}(\widetilde{t}, \widetilde{x}, \widetilde{y}), \widetilde{v}=v^{\prime}(\widetilde{t}, \widetilde{x}, \widetilde{y}, \widetilde{z}), \widetilde{p}=\widetilde{P}(\widetilde{x})+p^{\prime}(\widetilde{t}, \widetilde{x}, \widetilde{y}, \widetilde{z}),(2)$ где $\mathrm{u}^{\prime}, v^{\prime}, p^{\prime}$ - параметры возмущений. Основное невозмущенное течение определяется величинами: $\widetilde{u}=\widetilde{U}(\widetilde{y}), \widetilde{v}=0, \widetilde{p}=\widetilde{P}(\widetilde{x})$.

Подставляем уравнение (2) в (1) и проведем процедуру линеаризации. В итоге получим следующую систему уравнений для возмущенного потока.

$$
\begin{aligned}
& \frac{\partial \widetilde{u}^{\prime}}{\partial \widetilde{t}}+\widetilde{U} \frac{\partial \widetilde{u}^{\prime}}{\partial \widetilde{x}}+u^{\prime} \frac{\partial \widetilde{U}}{\partial \widetilde{y}}=-\frac{1}{\rho} \frac{\partial \widetilde{p}^{\prime}}{\partial \widetilde{x}}+v\left(\frac{\partial^{2} \widetilde{x}^{\prime}}{\partial \widetilde{x}^{2}}+\frac{\partial^{2} \widetilde{u}^{\prime}}{\partial \widetilde{y}^{2}}\right)-\frac{v}{K} \widetilde{u}^{\prime}, \\
& \frac{\partial \widetilde{v}^{\prime}}{\partial \widetilde{t}}+\widetilde{U} \frac{\partial \widetilde{v}^{\prime}}{\partial \widetilde{x}}=-\frac{1}{\rho} \frac{\partial \widetilde{p}^{\prime}}{\partial \widetilde{y}}+v\left(\frac{\partial^{2} \widetilde{v}^{\prime}}{\partial \widetilde{x}^{2}}+\frac{\partial^{2} \widetilde{v}^{\prime}}{\partial \widetilde{y}^{2}}\right)-\frac{v}{K} \widetilde{v}^{\prime}, \\
& \frac{\partial \widetilde{u}^{\prime}}{\partial \widetilde{x}}+\frac{\partial \widetilde{v}^{\prime}}{\partial \widetilde{y}}=0 .
\end{aligned}
$$

Метод малых возмущений предполагает, что возмущенные скорость и давление пропорциональны волне . Функция тока возмущенной скорости определяется из выражений:

$u^{\prime}=\frac{\partial \psi}{\partial \widetilde{y}}, v^{\prime}=-\frac{\partial \psi}{\partial \widetilde{y}}, \psi=\widetilde{\varphi}(\widetilde{y}) \exp [i(\widetilde{\alpha} \widetilde{x}-\widetilde{\beta} \widetilde{t})]$,

где $\varphi(y)-$ функция тока, $\widetilde{\beta}=\widetilde{\beta}_{r}+i \widetilde{\beta}_{i}, \widetilde{\beta}_{r}-$ круговая частота отдельного колебания, $\widetilde{\beta}_{i}-$ коэффициент нарастания возмущений. Подставим (4) в (3). После этой подстановки продифференцируем $x$-вую компоненту уравнения движения по $y$, а $y$-вою компоненту по $x$. После процедуры дифференцирования вычтем $y$-вую компоненту от $x$-вой. В ходе этих операций исключено давление. Далее проведем процедуру обезразмеривания полученного выражения. В итоге имеем:

$$
(U-c)\left(\varphi^{\prime \prime}-\alpha^{2} \varphi\right)-U^{\prime \prime} \varphi=-\frac{i}{\alpha \operatorname{Re}}\left(\varphi^{\prime \prime \prime \prime}-2 \alpha^{2} \varphi^{\prime \prime}+\alpha^{4} \varphi\right)-\frac{i}{\alpha M \operatorname{Re}}\left(\alpha^{2} \varphi-\varphi^{\prime \prime}\right) .
$$

$\mathrm{B}$ случае, когда $\mathrm{Da} \rightarrow \infty$ уравнение (5) преобразуется в классическое уравнение Орра-Зоммерфельда.

Для того чтобы определить критерий гидродинамической неустойчивости необходимо исследовать уравнение (5) на собственные значения. Граничные условия возмущенной скорости на стенке имеют вид:

$\left.\left(\frac{d \varphi}{\partial y} \pm \mathrm{Kn} \frac{d^{2} \varphi}{d y^{2}}\right)\right|_{y= \pm 1}=0$.

\section{Основное течение}

Для того чтобы рассчитать критерии гидродинамической неустойчивости в пористой среде по уравнению (5) с граничными условиями (6) необходимо знать профиль невозмущенной скорости. Профиль основного течения был получен в работе [6] и имеет следующий вид $U=\frac{\delta \cosh (M)-\cosh (M y)}{\delta \cosh (M)-1}$,

где

$\delta=1+\mathrm{Kn} M \tanh (M)$.

\section{Численный расчет критериев устойчивости}

Задача на собственные значения для уравнения (5) решалась методом коллокаций. Приближение Галеркина для амплитуды скорости взято в следующем виде [5]: $\varphi=\sum_{j=1}^{N} a_{j} f_{j}(y)$.

Пробные функции выбираются в следующем виде:

$f_{i}(y)=\left(1-y^{2}\right)\left(1-y^{2} /\left(1+\frac{4 \mathrm{Kn}}{-1-3 \mathrm{Kn}+4 j \mathrm{Kn}}\right)\right) y^{2(j-1)}$

или

$f_{j}(y)=\left(1-y^{2}\right)\left(1-y^{2} /\left(1+\frac{4 \mathrm{Kn}}{-1-3 \mathrm{Kn}+4 j \mathrm{Kn}}\right)\right) T_{2 j}(y)$,

где $T_{j}(y)$ - полиномы Чебышева первого рода, которые определяются из

$T_{0}(y)=1$,

$T_{1}(y)=y$,

$T_{j+1}(y)-2 \mathrm{y} T_{j}(y)+T_{j-1}(z)=0,(-1 \leq \mathrm{y} \leq 1)$.

Для верификации методики исследования уравнения (5) были проведены тестовые расчеты для плоского канала при течении чистой жидкости $(\mathrm{Da} \rightarrow \infty)$ и при $\mathrm{Kn}=0$. При этом профиль невозмущенной скорости определялся уравнением

$U=1-y^{2}$.

Расчеты показали, что $\mathrm{Re}_{\text {кр }}=5722$. Это соответствует [11].

Далее были проведено сравнение результатов расчета на основе уравнения (5) с данными, работы [8] для чистого потока $(\mathrm{Da} \rightarrow \infty)$ при учете проскальзывания. Результаты сравнения представлены на рис.1, который показывает хорошее согласование. 


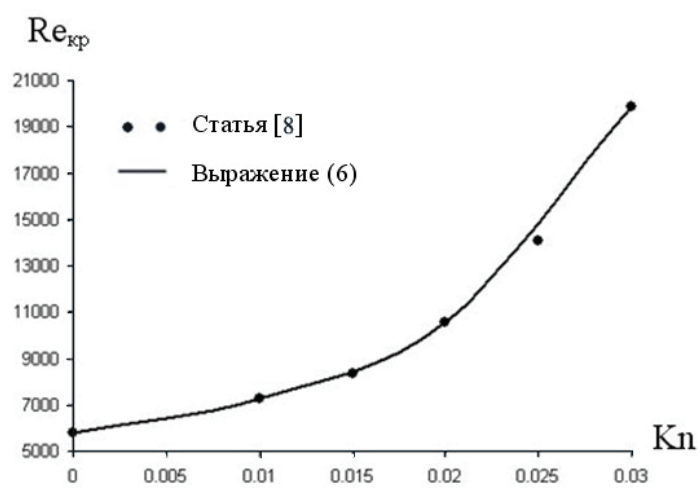

Рис. 1. Сравнение результатов расчета на основе (5) при $M=0$ и данных из работы [8].

Также результаты тестовых расчетов показали, что при $N=500$ отличие критических значений чисел Рейнольдса при использовании пробных функций $(11, a)$ и $(11$, б) составляет менее $0,3 \%$.

\section{Результаты расчета}

На основе предложенной математической модели были получены расчетные данные критических параметров устойчивости ламинарного течения разреженного потока в пористой среде при различных наборах значений параметра $M$ и числа Кнудсена.

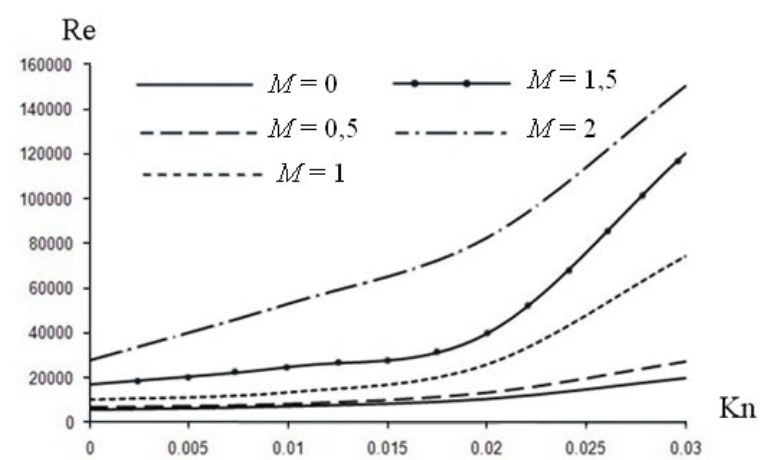

Рис. 2. Зависимость критического числа Рейнольдса от числа Кнудсена при разных значениях параметра $M$.

Из рисунка 2 видно, что увеличение числа Кнудсена и параметра $M$ приводит к стабилизации потока (критическое число Рейнольдса возрастает). Такой эффект обусловлен тем, что при увеличении $M$ и $\mathrm{Kn}$ увеличивается степень заполненности профиля скорости и это, в соответствии со второй теоремой Релея об устойчивости движения потока [10], ведет к стабилизации течения и к возрастанию значения критического числа Рейнольдса.

Из рис. 3 видно, что увеличение числа Кнудсена и параметра, учитывающего пористость $(M)$, приводит к уменьшению критического волнового числа. Это указывает на то, что критическая длина волны возмущения увеличивается. Т.е. длинноволновые возмущения являются более опасными для устойчивости потока.

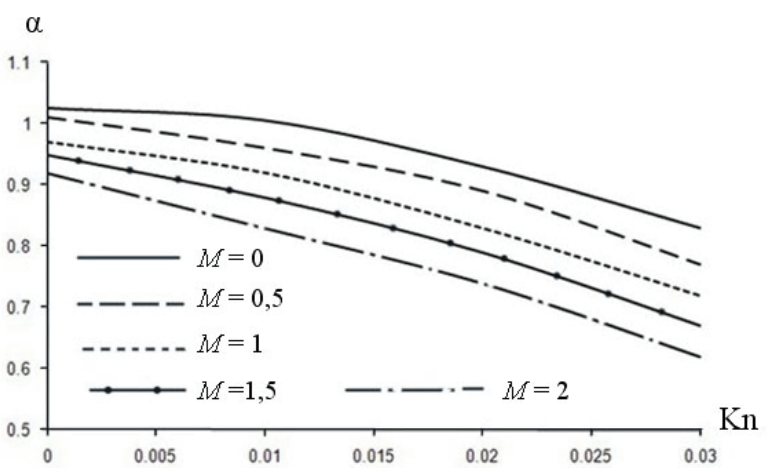

Рис. 3. Зависимость критического волнового числа от числа Кнудсена при разных значениях параметра $M$.

\section{Выводы}

Рассмотрена гидродинамическая неустойчивость разреженного потока в плоском канале, который заполнен пористой средой. Исследование проведено на основе выведенного уравнения движения для двухмерных возмущений. Уравнение учитывает линейную составляющую гидродинамического сопротивления пористой среды. На основе решения задачи на собственные значения методом коллокаций получена зависимость $\mathrm{Re}_{\text {кр }}=f(M, \mathrm{Kn})$. Расчеты показали, что при увеличении параметров $M$ и Кn поток становится более устойчивым и увеличивается критическая длина волны возмущения.

\section{ЛИТЕРАТУРА}

1. Коновалов Д.А., Дроздов И.Г., Лазаренко И.Н., Шматов Д.П. Моделирование процессов гидродинамики течения охладителя в наноструктурах на основе нитевидных кристаллов кремния // Вестник Воронежского государственного университета - 2013. - Т.9, №4. C. $1-8$.

2. Коновалов Д.А., Лазаренко И.Н, Кожухов Н.Н, Дроздов И.Г. Разработка методов интенсификации теплообмена в микроканальных теплообменниках гибридных систем термостабилизации // Вестник Воронежского государственного университета - 2016. - Т.12, №3. - C. $21-30$.

3. Jun Jie Liu, Hua Zhang, S. C. Yao, Yubai Li. Porous Media Modeling of Two-Phase Microchannel Cooling of Electronic Chips With Nonuniform Power Distribution // Journal of Electronic Packaging. - 2014. - V.136, №2. 021008.

4. Avrameko A.A., Tyrinov A.I., Shevchuk I.V., Dmitrenko N.P. Dean instability of nanofluids with radial temperature and concentration nonuniformity // Physic of fluids. -2016. - V.28. - P. 034104-1 - 0.4104-16.

5. Авраменко А.А., Тыринов А.И., Домамев В.Е., Ковецкая М.М., Сорокина Т.В., Дмитренко Н.П. Неустойчивость скользящего потока в криволинейном канале // Промышленная теплотехника. - 2013. - Т. 35, №2, C. $11-16$.

6. Nield D.A. The stability of flow in a channel or duct occupied by a porous medium // Int. J. Heat Transfer. - 
2003. - V. 46, №2. - P.4351 - 4354.

7. Avramenko A.A., Kuznetsov, A.V., Nield D.A. Instability of slip flow in a channel occupied by a hyperporous medium // Jornal of porous media - 2007. - V.10, №5 P. $435-442$.

8. Lauga E., Cossu C.A. A note on the stability of slip channel flows // Phys. Fluids. - $2005-$ V. 17, №8 P.088106-1 - 088106-4.

\section{INSTABILITY FLOW IN CHANNEL, OCCUPIED POROUS MEDIA}

\section{Avramenko A.A., Dmytrenko N.P., Kovetskaya U.U.}

Institute of Engineering Thermophysics of the National Academy of Sciences of Ukraine, vul. Zhelyabova, 2a, Kyiv, 03680 Ukraine.

The flow in porous media may be either laminar or turbulent. And it is important to know conditions and parameters at which one flow regime crosses into another. In this article consider the features of the slip flow dynamics in a porous medium, in order to determine the instability criterion. For this aim we use a two-dimensional formulation of the problem and collocation method.

The hydrodynamic instability of a rarefied flow in a flat channel, which is occupied porous medium, is considered. This study is based on the derived motion equation for twodimensional perturbations. The equation takes into account only the linear component of the porous medium. On the basis of the solving the problem on their own values by the collocation method we obtained relationship . Calculations have shown that the increasing of parameters $\mathrm{M}$ and $\mathrm{Kn}$ lead to stability flow and to the growth of the critical perturbation length.

References 11, figures 3.

Key words: instability, porosity, permeability, slip flow, perturbation number.

1. Konovalov D.A., Drozdov I.G., Lazarenko I.N., Shmatov D.P. Modelirovanie procesov gidrodinamiki techeniya ohladitelya $\mathrm{v}$ nanostrukturah na ocnove niteevidnuh kristalov kremniya [Simulation of coolant flow hydrodynamics processes in nanostructures based on silicon whiskers], Vestnik Voronezhskogo universiteta. 2013, T.9, №4, P.1 - 8. (Rus).
9. Gad-el-Hak M. The fluid mechanics of microdevices // J. Fluids Engineering. - 1999. - V. 121, №1 - P.5 - 33.

10. Schlichting $H$., Gersten $K$. Boundary Layer Theory. -8 th ed. Berlin. Springer: $-2000 .-799 p$.

11. Orsag S.A. Accurate solution of the Orr-Sommerfeld stability equitation // J. Fluid Mech. - 1971. - V.50, P. 689-703.

2. Konovalov D.A., Lazarenko I.N., Kozhuhov N.N., Drozdov I.G. Razrabotka metodov intensifikatsii teploobmena $\mathrm{v}$ microcanalnuh teploobmennikax gibridnuh system termostabilizatsii [Development of methods for enhancement of heat transfer in microchannel heat exchangers, thermal stabilization of hybrid systems], Vestnik Voronezhskogo universiteta. 2016, T.12, №3, P.2130. (Rus).

3. Jun Jie Liu, Hua Zhang, S. C. Yao, Yubai Li. Porous Media Modeling of Two-Phase Microchannel Cooling of Electronic Chips With Nonuniform Power Distribution, Journal of Electronic Packaging. 2014, V.136, №2, 021008.

4. Avrameko A.A., Tyrinov A.I., Shevchuk I.V., Dmitrenko N.P. Dean instability of nanofluids with radial temperature and concentration nonuniformity, Physic of fluids, 2016, V.28, P. 034104-1-0.4104-16.

5. Avrameko A.A., Tyrinov A.I., Domashev V.E., Kovetskaya M.M., Sorokina T.V., Dmitrenko N.P. Neustoichivost potoka $\mathrm{v}$ krivolineinom kanale [The instability of the moving flow in a curved channel] // Promyshlennaya teplotekhnika [Industrial Heat Engineering], 2013, T. 35, №2, P. 11-16. (Rus).

6. Nield D.A. The stability of flow in a channel or duct occupied by a porous medium, Int. J. Heat Transfer. 2003, V. 46, №2, P.4351-4354.

7. Avramenko A.A., Kuznetsov, A.V., Nield D.A. Instability of slip flow in a channel occupied by a hyperporous medium, Jornal of porous media, 2007, V.10, №5, P. 435-442.

8. Lauga E., Cossu C.A. A note on the stability of slip channel flows, Phys. Fluids. 2005, V. 17, №8, P.088106-1088106-4.

9. Gad-el-HakM. The fluid mechanics of microdevices, J. Fluids Engineering. 1999, V. 121, №1, P.5-33.

10. Schlichting H., Gersten K. Boundary Layer Theory. 8th ed. Berlin. Springer: 2000, 799p.

11. Orsag S.A. Accurate solution of the Orr-Sommerfeld stability equitation, J. Fluid Mech. 1971, V.50, P. 689-703. 